\title{
IONIZED NITROGEN AT HIGH REDSHIFT
}

\author{
R. Decarli ${ }^{1}$, F. Walter ${ }^{1,2}$, R. Neri ${ }^{3}$, F. Bertoldi ${ }^{4}$, C. Carilli ${ }^{5}$, P. Cox $^{3}$, J. P. Kneib ${ }^{6}$, J. F. Lestrade ${ }^{7}$, R. Maiolino ${ }^{8}$,

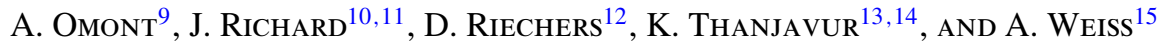 \\ ${ }^{1}$ Max-Planck Institut für Astronomie, Königstuhl 17, D-69117 Heidelberg, Germany; decarli@ mpia.de \\ ${ }^{2}$ NRAO, 520 Edgemont Road, Charlottesville, VA 22903-2475, USA \\ ${ }^{3}$ IRAM, 300 rue de la piscine, F-38406 Saint-Martin d'Hères, France \\ ${ }^{4}$ Argelander Institute for Astronomy, University of Bonn, Auf dem Hügel 71, D-53121 Bonn, Germany \\ ${ }^{5}$ NRAO, Pete V. Domenici Array Science Center, P.O. Box O, Socorro, NM 87801, USA \\ ${ }^{6}$ Laboratoire d'Astrophysique de Marseille, Observatoire d'Astronomie Marseille-Provence, BP 8, F-13376 Marseille, France \\ ${ }^{7}$ Observatoire de Paris, CNRS, 61 Av. de l'Observatoire, F-75014 Paris, France \\ ${ }^{8}$ INAF-Osservatorio Astronomico di Roma, via di Frascati 33, I-00040 Monte Porzio Catone, Italy \\ ${ }^{9}$ Institut d'Astrophysique de Paris, UPMC and CNRS, 98bis Bld. Arago, F-75014 Paris, France \\ ${ }^{10}$ CRAL, Observatoire de Lyon, Université Lyon 1, 9 Avenue Ch. André, F-69561 Saint Genis Laval Cedex, France \\ ${ }^{11}$ Dark Cosmology Centre, Niels Bohr Institute, University of Copenhagen, Juliane Maries Vej 30, DK-2100 Copenhagen, Denmark \\ ${ }_{12}$ Astronomy Department, Caltech, 1200 East California Boulevard, Pasadena, CA 91125, USA \\ ${ }^{13}$ Canada-France-Hawaii Telescope Corporation, HI 96743, USA \\ ${ }^{14}$ Department of Physics and Astronomy, University of Victoria, Victoria, BC V8P 1A1, Canada \\ 15 Max-Planck-Institut für Radioastronomie, Auf dem Hügel 69, D-53121 Bonn, Germany \\ Received 2011 December 21; accepted 2012 March 30; published 2012 May 18
}

\begin{abstract}
We present secure $\left[\mathrm{N} \mathrm{II}_{205 \mu \mathrm{m}}\right.$ detections in two millimeter-bright, strongly lensed objects at high redshift, APM 08279+5255 $(z=3.911)$ and MM $18423+5938(z=3.930)$, using the IRAM Plateau de Bure Interferometer. Due to its ionization energy $\left[\mathrm{N} \mathrm{II}_{205} \mu \mathrm{m}\right.$ is a good tracer of the ionized gas phase in the interstellar medium. The measured fluxes are $S\left([\mathrm{~N} \mathrm{II}]_{205 \mu \mathrm{m}}\right)=(4.8 \pm 0.8) \mathrm{Jy} \mathrm{km} \mathrm{s}^{-1}$ and $(7.4 \pm 0.5) \mathrm{Jy} \mathrm{km} \mathrm{s} \mathrm{k}^{-1}$, respectively, yielding line luminosities of $L\left(\left[\mathrm{~N} \mathrm{II}_{205} \mu \mathrm{m}\right)=(1.8 \pm 0.3) \times 10^{9} \mu^{-1} L_{\odot}\right.$ for APM $08279+5255$ and $L\left(\left[\mathrm{~N} \mathrm{II}_{205 \mu \mathrm{m}}\right)=\right.$ $(2.8 \pm 0.2) \times 10^{9} \mu^{-1} L_{\odot}$ for MM $18423+5938$. Our high-resolution map of the $\left[\mathrm{N}_{\mathrm{II}}\right]_{205} \mu \mathrm{m}$ and $1 \mathrm{~mm}$ continuum emission in MM 18423+5938 clearly resolves an Einstein ring in this source and reveals a velocity gradient in the dynamics of the ionized gas. A comparison of these maps with high-resolution EVLA CO observations enables us to perform the first spatially resolved study of the dust continuum-to-molecular gas surface brightness $\left(\Sigma_{\mathrm{FIR}} \propto \Sigma_{\mathrm{CO}}^{N}\right.$, which can be interpreted as the star formation law) in a high-redshift object. We find a steep relation $(N=1.4 \pm 0.2)$, consistent with a starbursting environment. We measure a [N II $]_{205 \mu \mathrm{m}} /$ FIR luminosity ratio in APM 08279+5255 and MM $18423+5938$ of $9.0 \times 10^{-6}$ and $5.8 \times 10^{-6}$, respectively. This is in agreement with the decrease of the $\left[\mathrm{N} \mathrm{II}_{205 \mu \mathrm{m}} /\right.$ FIR ratio at high FIR luminosities observed in local galaxies.
\end{abstract}

Key words: galaxies: individual (MM 18423+5938) - galaxies: ISM - quasars: individual (APM 08279+5255)

Online-only material: color figures

\section{INTRODUCTION}

Forbidden atomic fine-structure transitions are important cooling lines of the interstellar medium (ISM). They provide effective cooling in cold regions where allowed atomic transitions cannot be excited, and thus are critical diagnostic tools to study the star-forming ISM. Perhaps the most important cooling line is the forbidden ${ }^{2} P_{3 / 2} \rightarrow{ }^{2} P_{1 / 2}$ fine-structure line of ionized carbon ([C II $]$ ) at $158 \mu \mathrm{m}$, which alone accounts for $0.1 \%-1 \%$ of the total continuum far-infrared (FIR) luminosity in local, starforming galaxies (see, e.g., Malhotra et al. 2001). Other main cooling atomic lines are the oxygen [O I] $(63 \mu \mathrm{m}$ and $145 \mu \mathrm{m})$ and [O III] $(52 \mu \mathrm{m}$ and $88 \mu \mathrm{m})$ lines, as well as the nitrogen [N II] lines at $122 \mu \mathrm{m}$ and $205 \mu \mathrm{m}$.

As the ionization potential of carbon is $11.3 \mathrm{eV}$ (hydrogen: $13.6 \mathrm{eV}),[\mathrm{CII}]$ is a tracer for both the neutral atomic and ionized medium, predominantly of photon-dominated regions. The ionization potentials for oxygen and nitrogen, on the other hand, are $13.6 \mathrm{eV}$ and $14.5 \mathrm{eV}$, respectively, implying that their ions trace the ionized medium. The $[\mathrm{N} \mathrm{II}]_{205 \mu \mathrm{m}}$ transition is of particular interest as it has a critical density in the ionized medium that is very close to that of [C II], thus potentially providing complementary information on the origin of the [C II] emission (e.g., Oberst et al. 2006; Walter et al. 2009b).
In studies of star formation at high- $z$, the need for diagnostics other than [C II] is also motivated by two other reasons: (1) the relative intensities of [C $\mathrm{II}]$ and $\left[\mathrm{NII}_{205} \mu \mathrm{m}\right.$ are susceptible to N/C abundance variations (e.g., Matteucci \& Padovani 1993), thus acting as diagnostics of metal enrichment in the first galaxies. (2) The [C II] line falls out of the $1.3 \mathrm{~mm}$ window at $z \approx 8$ and will not be observable at $z$ up to 10.2 with ALMA, as no $2 \mathrm{~mm}$ receiver (ALMA "band 5") will be available for a large number of antennas. This limits [C II] studies in the first galaxies observable at the beginning of cosmic reionization.

Whereas the $[\mathrm{C} \mathrm{II}]$ line has now been abundantly detected in the local universe (e.g., Stacey et al. 1991; Madden et al. 1997; Luhman et al. 1998; Malhotra et al. 2001; Beirão et al. 2010; Edge et al. 2010; Fischer et al. 2010; Ivison et al. 2010; Loenen et al. 2010) and is now (almost) routinely detected at high redshift (Maiolino et al. 2005, 2009; Iono et al. 2006; Walter et al. 2009a; Wagg et al. 2010; Stacey et al. 2010; Cox et al. 2011; De Breuck et al. 2011), measurements of the $\left[\mathrm{N}_{\mathrm{II}}\right]_{205} \mu \mathrm{m}$ line are scarce. The $\left[\mathrm{N} \mathrm{III}_{205} \mu \mathrm{m}\right.$ line was first detected by FIRAS aboard COBE in the Milky Way (Wright et al. 1991), and later in the Galactic H II regions G333.6-0.2 Colgan et al. (1993) and DR21 (White et al. 2010) and in the Carina Nebula (Oberst et al. 2006). The $\left[\mathrm{N} \mathrm{II}_{205 \mu \mathrm{m}}\right.$ line has also been recently detected in some local galaxies, e.g., 
NGC 1097 (Beirão et al. 2010; see also Garciá-Carpio et al. 2011). On the other hand, little is known about nitrogen at high redshift. Multiple attempts were performed to detect this line at high redshift (4C41.17 and PC2047+0123: Ivison \& Harrison 1996; Cloverleaf: Benford 1999; APM 08279: Krips et al. 2007; J1148+5251: Walter et al. 2009b; SDP.81: Valtchanov et al. 2011). However, all these measurements, except the one on the $z=6.42$ quasar $\mathrm{J} 1148+5251$, were too insensitive by about an order of magnitude to potentially detect the $[\mathrm{N} \mathrm{II}]_{205} \mu \mathrm{m}$ line. Very recently, Ferkinhoff et al. (2011) reported the first detection of the second line of ionized nitrogen, $[\mathrm{N} \mathrm{II}]_{122 \mu \mathrm{m}}$, in two $z \sim 2.7$ targets, SMMJ02399-0136 $(z=2.81)$ and the Cloverleaf QSO $(z=2.56)$. Bradford et al. (2011) published a tentative $(1.5 \sigma)$ detection of $[\mathrm{N} \mathrm{II}]_{205 \mu \mathrm{m}}$ in the lensed quasar APM 08279+5255 $(z=3.911)$, and Combes et al. (2012) reported the detection of the $[\mathrm{N} \mathrm{II}]_{205 \mu \mathrm{m}}$ line in a lensed submillimeter galaxy (SMG), HLSJ091828.6+514223, at $z=5.2$.

Here we present secure detections of the $[\mathrm{N} \mathrm{II}]_{205 \mu \mathrm{m}}$ finestructure line in two high- $z$ sources, the lensed quasar APM $08279+5255(z=3.911)$ and the SMG MM 18423+5938 $(z=3.930)$. These sources are strongly magnified by gravitational lensing (magnification factor $\mu=4-100$ for APM 08279+5255 and 12-20 for MM 18423+5938; see Egami et al. 2000; Riechers et al. 2009; Lestrade et al. 2011), and represent two of the brightest molecular emitters at this redshift, with $\mathrm{CO}(6-5)$ peak flux densities of $7.3 \mathrm{mJy}$ (Weiß et al. 2007) and $33 \mathrm{mJy}$ (Lestrade et al. 2010), respectively. Observations were carried out at the IRAM Plateau de Bure Interferometer $(\mathrm{PdBI})$ and are summarized in Section 2. Results are presented in Section 3.

Throughout the paper we will assume a standard cosmology with $H_{0}=70 \mathrm{~km} \mathrm{~s}^{-1} \mathrm{Mpc}^{-1}, \Omega_{\mathrm{m}}=0.3$, and $\Omega_{\Lambda}=0.7$.

\section{OBSERVATIONS}

In our observations we exploited the capabilities of the new "band 4" receiver at PdBI. Covering the frequency range between 277 and $371 \mathrm{GHz}$ (with a small gap due to atmospheric absorption at roughly $320-330 \mathrm{GHz}$ ), this band opens up the opportunity to search for the $[\mathrm{N} \mathrm{III}]_{205 \mu \mathrm{m}}$ line in a wide redshift range $(2.85<z<4.27)$.

APM $08279+5255$ was observed in compact array configuration with five antennas (6Cq-E10) on 2011 March 8. Baselines ranged between 20 and $140 \mathrm{~m}$. 3C273, 3C84, and MWC349 were used as amplitude calibrators. The quasar $0917+624$ was observed every 30 minutes for phase calibration. The time on sources was $4.5 \mathrm{hr}$ (five-antenna equivalent). MM 18423+5938 was observed in both compact and extended configurations (6Cq-E10 and 6Bq) between 2011 January 3 and 2011 March 9. Baselines ranged between 17 and $446 \mathrm{~m}$. 3C273, 3C 345, MWC349, and 3C84 were used as amplitude calibrators, while $1849+670$ was observed as phase calibrator. The total time on source was $10 \mathrm{hr}$ (six-antenna equivalent).

The tuning frequencies were 297.522 and $296.400 \mathrm{GHz}$, respectively, based on the $\mathrm{CO}$ redshift of the sources (Weiß et al. 2007; Lestrade et al. 2010, 2011). The receiver worked in lower-side band. System temperature ranged between 150 and $350 \mathrm{~K}$. Data reduction and analysis were performed using the most recent version of the GILDAS package. Maps were extracted using natural weighting. This allows us to fully recover the flux of our sources, given that their spatial extent $\left(\lesssim 2^{\prime \prime}\right)$ is comparable with or smaller than the angular scale filtered in by the smallest baselines in our observations $\left(\sim 2^{\prime \prime}\right)$. The resulting synthesized beams are $1^{\prime \prime} .2 \times 0.9$ for APM $08279+5255$ and
1.. $0 \times 0$ ". 9 for MM 18423+5938. In order to take full advantage of the high spatial resolution observations of MM 18423+5938, we also extracted a pure continuum map of this source using uniform weighting. This high-resolution map has a synthetic beam size of $0.6 \times 0$.' 5 , at the price of filtering out a significant fraction $(\sim 66 \%)$ of the flux from the extended emission of the object. Therefore, in the remainder of the analysis, all the flux measurements will refer only to the map obtained with natural weighting. The $1 \sigma$ noise per $20 \mathrm{MHz}$ wide channel $\left(\approx 20.2 \mathrm{~km} \mathrm{~s}^{-1}\right)$ is $3.4 \mathrm{mJy}$ for APM $08279+5255$ and $2.4 \mathrm{mJy}$ for $\mathrm{MM} 18423+5938$, corresponding to a $1 \sigma$ sensitivity of $0.66 \mathrm{mJy} \mathrm{beam}^{-1}$ and $0.82 \mathrm{mJy}^{\text {beam }}{ }^{-1}$ over the line width (see Section 3).

\section{RESULTS}

\section{1. [N II $]_{205 \mu m}$ and Continuum Emission}

\subsubsection{APM $08279+5255$}

Figure 1 shows the observed spectrum and the (continuumsubtracted) line map of APM 08279+5255. The $[\mathrm{N} \mathrm{II}]_{205} \mu \mathrm{m}$ line is detected at modest significance. We fitted the spectrum with a flat continuum plus a Gaussian profile for the $[\mathrm{N} \mathrm{II}]_{205 \mu \mathrm{m}}$ emission. The fitted $[\mathrm{N} \mathrm{II}]_{205 \mu \mathrm{m}}$ flux is $S\left([\mathrm{~N} \mathrm{II}]_{205 \mu \mathrm{m}}\right)=4.8 \pm$ $0.8 \mathrm{Jy} \mathrm{km} \mathrm{s}^{-1}$, consistent with the tentative detection $(7.6 \pm$ $5.4 \mathrm{Jy} \mathrm{km} \mathrm{s}^{-1}$ ) reported in Bradford et al. (2011). We measure a line width of $570 \pm 110 \mathrm{~km} \mathrm{~s}^{-1}$, consistent with the weighted average of the line width values from $\mathrm{CO}$ transitions reported in Weiß et al. (2007). Line luminosities $L_{c}$ and $L^{\prime}$ are derived following Solomon et al. (1992), and reported in Table 1 together with all the relevant numbers and fitted parameters. We measure a continuum flux of $33 \pm 3 \mathrm{mJy}$, in agreement with the extrapolation between the SCUBA and PdBI observations at $850 \mu \mathrm{m}$ and $1.4 \mathrm{~mm}$, respectively (Weiß et al. 2007).

\subsection{2. $M M 18423+5938$}

In Figure 2, we show the integrated spectrum of the $[\mathrm{N} \mathrm{II}]_{205 \mu \mathrm{m}}$ emission in MM 18423+5938. The line is detected at very high significance. The Gaussian fit gives an integrated $[\mathrm{N} \mathrm{II}]_{205 \mu \mathrm{m}}$ flux of $S\left([\mathrm{~N} \mathrm{II}]_{205 \mu \mathrm{m}}\right)=7.4 \pm 0.5 \mathrm{Jy} \mathrm{km} \mathrm{s}^{-1}$ and a line width of $230 \pm 20 \mathrm{~km} \mathrm{~s}^{-1}$ (for a comparison, the $\mathrm{CO}(1-0)$ and $\mathrm{CO}(2-1)$ lines reported in Lestrade et al. 2011 have widths of $160 \pm 30$ and $240 \pm 30 \mathrm{~km} \mathrm{~s}^{-1}$, respectively). Another line is tentatively detected at $298.23 \pm 0.02 \mathrm{GHz}$, i.e., at rest frequency $1470.22 \pm 0.11 \mathrm{GHz}$, consistent with three different transitions of sulfur dioxide, $\mathrm{SO}_{2}$ (at 1470.225, 1470.327, and 1470.342 $\mathrm{GHz}$, respectively). Given the low signal-to-noise ratio $(\mathrm{S} / \mathrm{N})$ of this line $(\sim 3.5 \sigma)$, we fitted it with a Gaussian by imposing the same line width as observed for $[\mathrm{N} \mathrm{II}]_{205 \mu \mathrm{m}}$. We find a continuum flux (integrated over the spatial extension of the emission) of $53 \pm 2 \mathrm{mJy}$, consistent with the $1.2 \mathrm{~mm}$ MAMBO flux reported in Lestrade et al. (2010), assuming a graybody dust with opacity index $\beta=1$.

Our PdBI observations clearly resolved the $1 \mathrm{~mm}$ continuum emission of MM 18423+5938. The Einstein ring reported in Lestrade et al. (2011) is clearly seen in the pure-continuum, high-resolution map shown in Figure 3 (top-left panel). Also the $[\mathrm{N} \mathrm{II}]_{205 \mu \mathrm{m}}$ emission appears clearly extended even in the lower resolution maps shown in the top-right panel of Figure 3. The $[\mathrm{N} \mathrm{II}]_{205} \mu \mathrm{m}$ line emission shows a clear velocity gradient from northeast (redshifted) to southwest (blueshifted). This is highlighted in Figure 3 (bottom-left panel) where we overplot the maps of the red and blue wings of the $[\mathrm{N} \mathrm{II}]_{205 \mu \mathrm{m}}$ line. We 

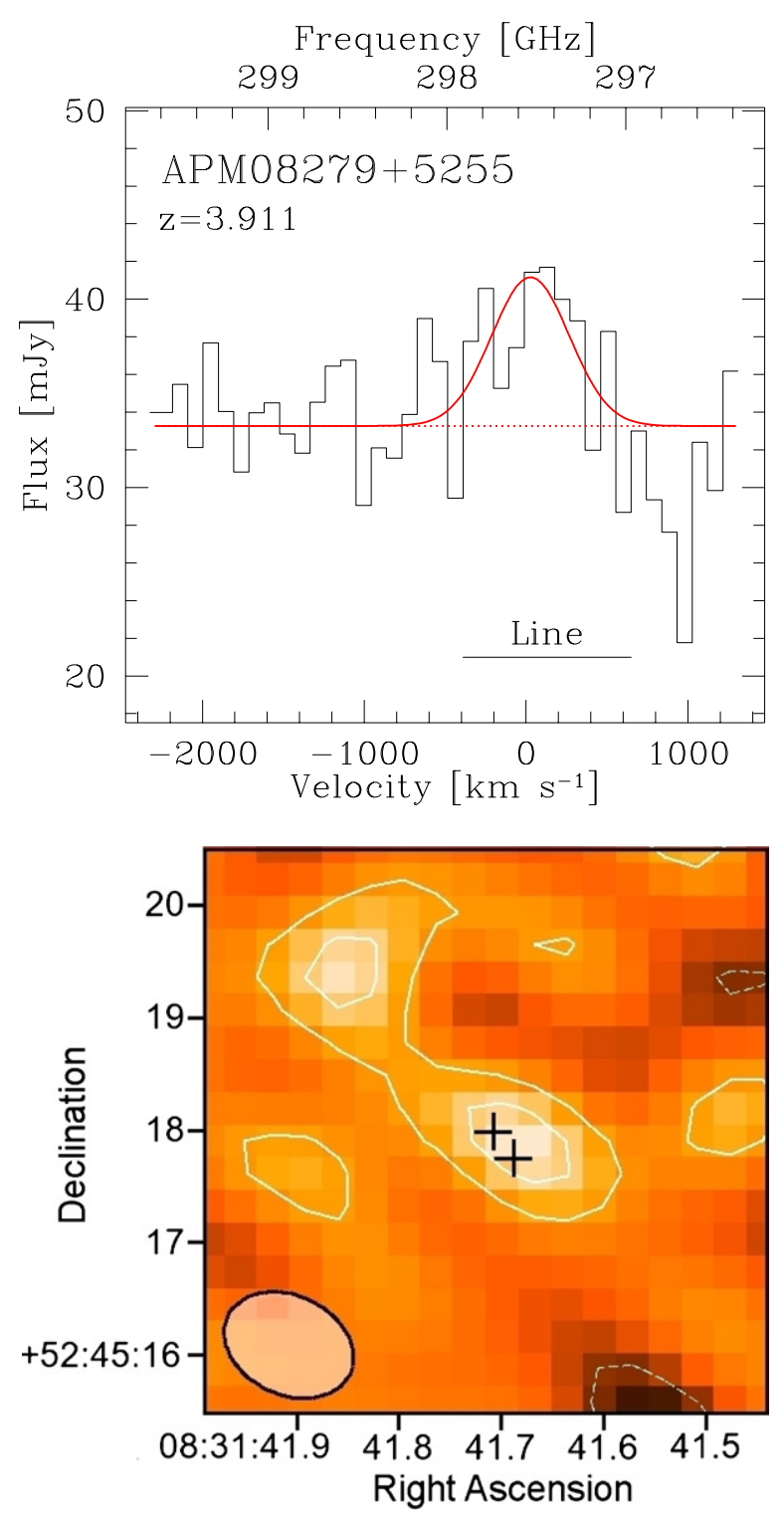

Figure 1. Observed spectrum (top) and continuum-subtracted map (bottom) of the $[\mathrm{N} \mathrm{II}]_{205 \mu \mathrm{m}}$ emission in APM 08279+5255. The spectrum is resampled into $95 \mathrm{~km} \mathrm{~s}^{-1}$ wide bins. The velocity scale is set assuming the CO redshift, $z=$ 3.911. The continuum and line fits are shown with thick, solid red lines. The channels used to create the $\left[\mathrm{N} \mathrm{II}_{205} \mu \mathrm{m}\right.$ line map are marked by a horizontal line. In the map, solid thick (dashed thin) contours are positive (negative) isophotes, separated by $2 \sigma\left(1 \sigma=0.47 \mathrm{mJy}\right.$ beam $\left.^{-1}\right)$. The synthetic beam $\left(1^{\prime \prime} .2 \times 00^{\prime \prime} 9\right)$ is shown in the bottom-left corner. The two crosses mark the position of the two lensed images reported by Riechers et al. (2009).

(A color version of this figure is available in the online journal.)

measure peak-to-peak velocity differences of $\sim 180 \mathrm{~km} \mathrm{~s}^{-1}$ and a velocity dispersion $\sim 80 \mathrm{~km} \mathrm{~s}^{-1}$. The $v / \sigma>1$ value indicates ordered kinematics for the ionized gas in the lensed source. A detailed model of the lens is required to reconstruct the intrinsic brightness of the source from these images and to properly constrain the dynamics of the system. This is beyond the scope of this paper.

\section{2. [N II $]_{205 \mu m}$ and $\mathrm{CO}$}

Here, we compare our $[\mathrm{N} \mathrm{II}]_{205 \mu \mathrm{m}}$ observations with the available literature data on $\mathrm{CO}$ emission in the two targets. In particular, the $[\mathrm{N} \mathrm{II}]_{205 \mu \mathrm{m}}$ to $\mathrm{CO}(6-5)$ luminosity ratio in our sources $\left([\mathrm{N} \mathrm{III}]_{205 \mu \mathrm{m}} / \mathrm{CO}(6-5)=1.5 \pm 0.4\right.$ in APM $08279+5255$

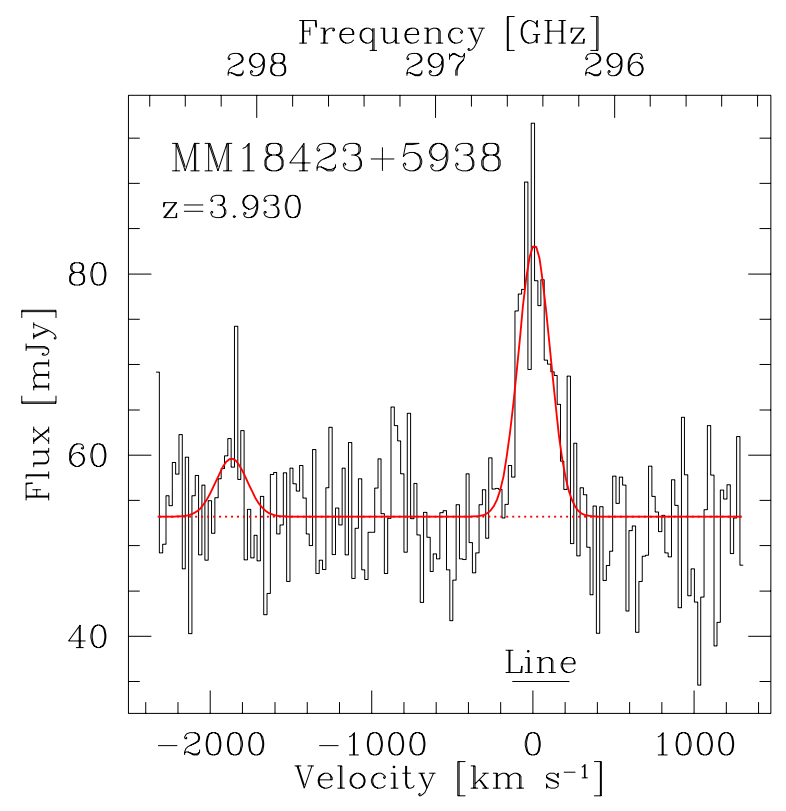

Figure 2. Integrated spectrum of the $\left[\mathrm{N} \mathrm{II}_{205} \mu \mathrm{m}\right.$ plus continuum emission in MM 18423+5938. Data are resampled into $20 \mathrm{~km} \mathrm{~s}^{-1}$ wide bins. The continuum and line fits are shown with thick, solid red lines. The tentative $\mathrm{SO}_{2}$ detection at $298.23 \mathrm{GHz}$ is also fitted by forcing the same line width as observed for $[\mathrm{N} \mathrm{II}]_{205} \mu \mathrm{m}$. The channels used to create the $[\mathrm{N} \mathrm{II}]_{205} \mu \mathrm{m}$ line map are marked by a horizontal line.

(A color version of this figure is available in the online journal.)

and $2.3 \pm 0.3$ in $\mathrm{MM} 18423+5938)$ is similar to the values reported by White et al. (2010) in the Galactic region DR21 ([N II $\left.]_{205 \mu \mathrm{m}} / \mathrm{CO}(6-5)=1.26 \pm 0.35\right)$, by Panuzzo et al. (2010) in M82 ([N II $\left.]_{205 \mu \mathrm{m}} / \mathrm{CO}(6-5)=1.68 \pm 0.05\right)$ and by van der Werf et al. (2010) in Mrk231 ([N II $]_{205 \mu \mathrm{m}} / \mathrm{CO}(6-5) \sim 1.2$ ).

In Figure 3 (bottom-right panel) we compare our [N II $]_{205 \mu \mathrm{m}}$ map of MM $18423+5938$ with our map of the $1 \mathrm{~mm}$ continuum emission and the $\mathrm{CO}(2-1)$ observations presented in Lestrade et al. (2011), which have similar $\mathrm{S} / \mathrm{N}$ and spatial resolution as the $\left[\mathrm{N} \mathrm{II}_{205} \mu \mathrm{m}\right.$ data presented here. Since nitrogen emission is tracing the ionized gas, while the $\mathrm{CO}$ emission maps the molecular gas (which is the fuel of star formation) and the FIR continuum traces the distribution of dust heated by young stars, this comparison allows for a direct, spatially resolved study of three important components of the ISM that are related to star formation. From the figure, it is apparent that the emission from the three tracers shows different morphologies (e.g., most of $[\mathrm{N} \mathrm{II}]_{205 \mu \mathrm{m}}$ and $\mathrm{CO}(2-1)$ emission arises in two blobs in the east and southwest parts of the Einstein ring, while a bright continuum emission is observed also on the northern side of the ring).

More quantitatively, in Figure 4 we perform a pixel-bypixel comparison of the emission of the FIR continuum with respect to the $[\mathrm{N} \mathrm{II}]_{205 \mu \mathrm{m}}$ and $\mathrm{CO}(2-1)$ lines. ${ }^{16}$ In order to avoid oversampling, we consistently rebinned all the maps into $0.5 \times 0.5$ pixels, and considered only pixels with $>1 \sigma$ flux in each axis. We find that the surface brightness of the continuum, $\Sigma_{\text {FIR }}$, shows a steep correlation with the surface brightnesses of $\left[\mathrm{N}_{\mathrm{II}}\right]_{205 \mu \mathrm{m}}$ and $\mathrm{CO}(2-1), \Sigma_{[\mathrm{NII}]}$ and $\Sigma_{\mathrm{CO}}: \Sigma_{\mathrm{FIR}} \propto \Sigma_{[\mathrm{NII}]}^{1.6 \pm 0.1}$ and $\Sigma_{\text {FIR }} \propto \Sigma_{\text {CO }}^{1.4 \pm 0.2}$. The latter relation is of particular interest, as $\Sigma_{\text {FIR }}$ can be used as a proxy for the star formation surface density,

\footnotetext{
16 A continuum map obtained with natural weighting is used here, so that the beam size of the three maps (FIR continuum, [N $\mathrm{NI}_{205} \mu \mathrm{m}$, and $\mathrm{CO}(2-1)$ ) are similar.
} 
Table 1

Line and Continuum Properties in APM 08279+5255 and MM 18423+5938

\begin{tabular}{|c|c|c|c|c|}
\hline Quantity & Units & APM 08279+5255 & MM $18423+5938$ & References \\
\hline Redshift & & 3.911 & 3.930 & 1,2 \\
\hline$D_{\mathrm{L}}$ & $(\mathrm{Gpc})$ & 34.897 & 35.097 & \\
\hline$\mu$ & & $4-100$ & $12-20$ & $3,4,2$ \\
\hline$v_{\mathrm{obs}}$ & $(\mathrm{GHz})$ & 297.522 & 296.400 & 0 \\
\hline $1 \sigma \mathrm{rms}(20 \mathrm{MHz})$ & $\left(\mathrm{mJy}\right.$ beam $\left.^{-1}\right)$ & 3.4 & 2.4 & 0 \\
\hline \multicolumn{5}{|c|}{$[\mathrm{N} \mathrm{III}]_{205 \mu \mathrm{m}}$} \\
\hline$S\left([\mathrm{~N} \mathrm{II}]_{205 \mu \mathrm{m}}\right)$ & $\left(\mathrm{Jy} \mathrm{km} \mathrm{s}{ }^{-1}\right)$ & $4.8 \pm 0.8$ & $7.4 \pm 0.5$ & 0 \\
\hline FWHM & $\left(\mathrm{km} \mathrm{s}^{-1}\right)$ & $570 \pm 110$ & $230 \pm 20$ & 0 \\
\hline$L_{\mathrm{c}}\left([\mathrm{N} \mathrm{II}]_{205 \mu \mathrm{m}}\right)$ & $\left(10^{9} \mu^{-1} L_{\odot}\right)$ & $1.8 \pm 0.3$ & $2.8 \pm 0.2$ & 0 \\
\hline$L^{\prime}\left([\mathrm{N} \mathrm{II}]_{205} \mu \mathrm{m}\right)$ & $\left(10^{10} \mu^{-1} \mathrm{~K} \mathrm{~km} \mathrm{~s}^{-1} \mathrm{pc}^{2}\right)$ & $1.81 \pm 0.3$ & $2.8 \pm 0.2$ & 0 \\
\hline$L_{\mathrm{c}}\left([\mathrm{N} \mathrm{II}]_{205 \mu \mathrm{m}}\right) / L_{\mathrm{FIR}}$ & $\left(10^{-6}\right)$ & $9.0 \pm 1.5$ & $5.8 \pm 0.4$ & 0 \\
\hline$M(\mathrm{H} \mathrm{II})$ & $\left(10^{9} \mu^{-1} M_{\odot}\right)$ & $\gtrsim 4.1$ & $\gtrsim 6.4$ & 0 \\
\hline$M(\mathrm{H} \mathrm{II}) / M\left(\mathrm{H}_{2}\right)$ & & $\gtrsim 0.8 \%$ & $\gtrsim 2.9 \%$ & 0 \\
\hline \multicolumn{5}{|c|}{ Other lines } \\
\hline$L_{\mathrm{c}}\left([\mathrm{CI}]_{1-0}\right)$ & $\left(10^{9} \mu^{-1} L_{\odot}\right)$ & $0.118 \pm 0.016$ & $0.29 \pm 0.06$ & 5,2 \\
\hline$L_{\mathrm{c}}\left([\mathrm{CI}]_{2-1}\right)$ & $\left(10^{9} \mu^{-1} L_{\odot}\right)$ & $<0.23$ & $0.88 \pm 0.17$ & 6,2 \\
\hline$L_{\mathrm{c}}(\mathrm{CO}(1-0))$ & $\left(10^{9} \mu^{-1} L_{\odot}\right)$ & $0.0050 \pm 0.0004$ & $0.0129 \pm 0.0017$ & 3,7 \\
\hline$L_{\mathrm{c}}(\mathrm{CO}(2-1))$ & $\left(10^{9} \mu^{-1} L_{\odot}\right)$ & $0.048 \pm 0.011$ & $0.179 \pm 0.018$ & 3,7 \\
\hline$L_{\mathrm{c}}(\mathrm{CO}(3-2))$ & $\left(10^{9} \mu^{-1} L_{\odot}\right)$ & $0.196 \pm 0.018$ & $\ldots$ & 8 \\
\hline$L_{\mathrm{c}}(\mathrm{CO}(4-3))$ & $\left(10^{9} \mu^{-1} L_{\odot}\right)$ & $0.44 \pm 0.02$ & $0.59 \pm 0.06$ & 1,2 \\
\hline$L_{\mathrm{c}}(\mathrm{CO}(6-5))$ & $\left(10^{9} \mu^{-1} L_{\odot}\right)$ & $1.2 \pm 0.2$ & $1.19 \pm 0.11$ & 1,2 \\
\hline$L_{\mathrm{c}}(\mathrm{CO}(7-6))$ & $\left(10^{9} \mu^{-1} L_{\odot}\right)$ & $\ldots$ & $0.82 \pm 0.10$ & 2 \\
\hline$L_{\mathrm{c}}(\mathrm{CO}(9-8))$ & $\left(10^{9} \mu^{-1} L_{\odot}\right)$ & $3.16 \pm 0.16$ & $\ldots$ & 1 \\
\hline$L_{\mathrm{c}}(\mathrm{CO}(10-9))$ & $\left(10^{9} \mu^{-1} L_{\odot}\right)$ & $3.5 \pm 0.6$ & $\ldots$ & 1 \\
\hline$L_{\mathrm{c}}(\mathrm{CO}(11-10))$ & $\left(10^{9} \mu^{-1} L_{\odot}\right)$ & $3.7 \pm 0.6$ & $\ldots$ & 1 \\
\hline$L_{\mathrm{c}}(\mathrm{CO}(12-11))$ & $\left(10^{9} \mu^{-1} L_{\odot}\right)$ & $3.5 \pm 1.8$ & $\ldots$ & 9 \\
\hline$L_{\mathrm{c}}\left(\mathrm{SO}_{2}\right)$ & $\left(10^{9} \mu^{-1} L_{\odot}\right)$ & $\ldots$ & $0.60 \pm 0.16$ & 0 \\
\hline$M\left(\mathrm{H}_{2}\right)$ & $\left(10^{11} \mu^{-1} M_{\odot}\right)$ & $5.3 \pm 0.5$ & $2.2 \pm 0.3$ & 3,7 \\
\hline \multicolumn{5}{|c|}{ Continuum } \\
\hline$S(850 \mu \mathrm{m})$ & (mJy) & $75 \pm 4$ & $\ldots$ & 1 \\
\hline$S(1 \mathrm{~mm})$ & (mJy) & $33 \pm 3$ & $53 \pm 2$ & 0 \\
\hline$S(1.3 \mathrm{~mm})$ & (mJy) & $16.9 \pm 2.5$ & $30 \pm 2$ & 1,2 \\
\hline$L_{\mathrm{FIR}}$ & $\left(10^{13} \mu^{-1} L_{\odot}\right)$ & 20 & 48 & 1,2 \\
\hline
\end{tabular}

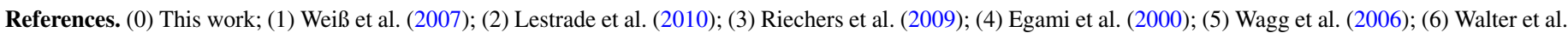
(2011); (7) Lestrade et al. (2011); (8) Downes et al. (1999); (9) Bradford et al. (2011).

$\Sigma_{\text {SFR }}$ (Kennicutt 1998), while $\Sigma_{\text {CO }}$ traces the surface density of the molecular gas, $\Sigma_{\mathrm{H}_{2}}$, which is the fuel for star formation. The right-hand panel of Figure 4 represents therefore the first spatially resolved study of the star formation law in a high- $z$ galaxy.

Locally, star formation surface density scales, to first order, linearly with the molecular gas surface density $\left(\Sigma_{\mathrm{SFR}} \propto \Sigma_{\mathrm{H}_{2}}^{N}\right.$, with $N \approx 1$; see, e.g., Bigiel et al. 2008; Leroy et al. 2008; Schruba et al. 2011). The relation steepens if one considers high-density environments and molecule-rich galaxies (e.g., Kennicutt 1998; Wong \& Blitz 2002; Daddi et al. 2010; Genzel et al. 2011). This appears to be the case for MM 18423+5938, where we measure a slope significantly larger than $1(N=$ $1.4 \pm 0.2)$. The steep relation observed between $\Sigma_{\mathrm{FIR}}$ and $\Sigma_{\left[\mathrm{N}_{\mathrm{II}}\right]}$ may indicate that, as star formation rate (SFR) increases, the ionization state of nitrogen changes, with an increasing fraction of multiply ionized $\mathrm{N}$ in the regions of most intense star formation. Moreover, if we divide the Einstein ring of MM 18423+5938 into two parts, north and south, with a cut at declination $+59: 38: 29.3$ (roughly corresponding to the center of the ring), we find that each part of the ring follows different power laws. The northern part of the ring has a $\sim 2$ times brighter continuum for a given $[\mathrm{NII}]_{205} \mu \mathrm{m}$ or $\mathrm{CO}(2-1)$ emission. The $\Sigma_{\mathrm{FIR}} \propto \Sigma_{\mathrm{CO}}^{N}$ relation shows a marginally flatter slope $(N=1.2)$ in the northern part than in the southern part $(N=1.6)$, in agreement with the relatively higher molecular content in the southern part of the ring.

\subsection{Ionized and Molecular Gas Masses}

Following Ferkinhoff et al. (2011), we can compute the minimum mass of ionized hydrogen in the high-density, hightemperature limit, assuming that all nitrogen in the $\mathrm{H}$ II regions is singly ionized:

$$
\begin{aligned}
M_{\min }(\mathrm{H} \mathrm{II}) & =\frac{L\left(\left[\mathrm{~N} \mathrm{II}_{205 \mu \mathrm{m}}\right) m_{\mathrm{H}}\right.}{\left(g_{1} / g\right) A_{10} h \nu_{10} \chi\left(\mathrm{N}^{+}\right)} \\
& \approx 2.27 \frac{L\left([\mathrm{~N} \mathrm{II}]_{205 \mu \mathrm{m}}\right)}{L_{\odot}} M_{\odot},
\end{aligned}
$$

where $A_{10}$ is the Einstein $A$ coefficient of the ${ }^{3} P_{1} \rightarrow{ }^{3} P_{0}$ transition of nitrogen $\left(2.08 \times 10^{-6} \mathrm{~s}^{-1}\right) ; g_{1}=3$ is the statistical weight of the $J=1$ level; $g=\Sigma_{i} g_{i} \exp \left(-\Delta E_{i} / k_{b} T\right)$ is the partition function, with $\Delta E_{i}$ being the energy difference between the fundamental and the $i$ th level, $k_{b}$ being the Boltzmann constant, and $T$ being the gas temperature; $h$ is Planck's constant; $v_{10}=1461.1318 \mathrm{GHz}$ is the rest-frame frequency of the transition; $m_{\mathrm{H}}$ is the mass of a hydrogen atom; and 



Figure 3. Top left: high-resolution (i.e., uniform-weighted) map of the pure continuum emission in MM 18423+5938. The Einstein ring is clearly resolved. Contours show the $+4 \sigma,+8 \sigma,+12 \sigma,+16 \sigma$ isophotes $\left(1 \sigma=0.58 \mathrm{mJy}_{\text {beam }}{ }^{-1}\right)$. The synthetic beam $\left(0{ }^{\prime \prime} 6 \times 00^{\prime \prime} 5\right)$ is also shown in the bottom-left corner. Top right: continuumsubtracted map of the $\left[\mathrm{N} \mathrm{II}_{205 \mu \mathrm{m}}\right.$ emission. Natural weighting is used here (beam size: $\left.1^{\prime \prime} .0 \times 0^{\prime \prime} 9\right)$. Contours are separated by $2 \sigma\left(1 \sigma=0.82 \mathrm{mJy}\right.$ beam $\left.{ }^{-1}\right)$. Bottom left: continuum-subtracted maps of the red and blue wings of the $\left[\mathrm{N} \mathrm{III}_{205 \mu \mathrm{m}}\right.$ emission in MM 18423+5938. The two sides of the line peak on opposite sides of the Einstein ring, unveiling a velocity gradient in the source. The contours from the continuum map in the top-right panel are also shown for reference. Bottom right: comparison of the $[\mathrm{N} \mathrm{II}]_{205 \mu \mathrm{m}}$ line map (white contours), the continuum emission at $1 \mathrm{~mm}$ (black contours), and the $\mathrm{CO}(2-1)$ map published by Lestrade et al. (2011) (color scale). The continuum emission is brighter on the eastern and northern sides of the ring, while $\mathrm{CO}$ and $[\mathrm{N} \mathrm{II}]_{205} \mu \mathrm{m}$ emission peaks in the east and southwest.

(A color version of this figure is available in the online journal.)

$\chi\left(\mathrm{N}^{+}\right)$is the $\mathrm{N}^{+} / \mathrm{H}^{+}$abundance ratio. In our working assumption, $\chi\left(\mathrm{N}^{+}\right)=\chi(\mathrm{N}) \approx 9.3 \times 10^{-5}$ (Savage \& Sembach 1996). This gives $M_{\min }\left(\mathrm{H}_{\mathrm{II}}\right)=4.1 \times 10^{9} \mu^{-1} M_{\odot}$ and $6.4 \times 10^{9} \mu^{-1} M_{\odot}$ for APM $08279+5255$ and MM $18423+5938$, respectively. It is interesting to compare these numbers with the molecular gas mass estimated from the $\mathrm{CO}(1-0)$ luminosity (Riechers et al. 2009; Lestrade et al. 2011): the minimum mass of the ionized gas is only a tiny fraction $(0.8 \%$ and $2.9 \%$, respectively) of the molecular reservoir in the two targets. Alternatively, the actual $\mathrm{N}^{+} / \mathrm{H}^{+}$abundance ratio may be significantly lower than the adopted value. This is likely to happen if these systems are metal poor (but this scenario is ruled out by the bright $\mathrm{CO} / \mathrm{FIR}$ luminosity ratios observed in our targets), or if the ISM is enshrouded in a hard radiation field (i.e., nitrogen is multiply ionized). This is likely happening in APM 08279+5255, which hosts a quasar (while no obvious signature of nuclear activity is observed in MM 18423+5938). Indeed, Ferkinhoff et al. (2010) estimated that an ionized gas mass of $\sim 3 \times 10^{9} M_{\odot}$ is present in APM 08279+5255, based on the [O III $]_{88 \mu \mathrm{m}}$ line emission. These observations are complementary to ours, as $\mathrm{N}$ is likely to be multiply ionized in the regions where most of the [O III] emission takes place.

\section{4. [N II $]_{205 \mu m}$ Contribution to ISM Cooling}

Finally, we evaluate the role of $\left[\mathrm{N}_{\mathrm{II}}\right]_{205 \mu \mathrm{m}}$ in the ISM cooling by comparing the $[\mathrm{N} \mathrm{II}]_{205 \mu \mathrm{m}}$ luminosity to that of the FIR continuum. The latter is taken from the spectral energy distribution fits by Weiß et al. (2007) for APM 08279+5255 $\left(L_{\mathrm{FIR}}=20 \times 10^{13} \mu^{-1} L_{\odot}\right),{ }^{17}$ and by Lestrade et al. (2010) for MM $18423+5938\left(L_{\mathrm{FIR}}=48 \times 10^{13} \mu^{-1} L_{\odot}\right)$. The observed $[\mathrm{N} \mathrm{II}]_{205 \mu \mathrm{m}} /$ FIR luminosity ratio is $9.0 \times 10^{-6}$ and $5.8 \times 10^{-6}$ in the two sources. In Figure 5, we compare these values with the measurements available in the literature. Since the majority of data refer to the $\left[\mathrm{N}_{\mathrm{II}}\right]_{122 \mu \mathrm{m}}$ transition, we converted our estimates assuming a [N II] 122-to-205 $\mu \mathrm{m}$ luminosity ratio of 5 (see, e.g., Beirão et al. 2010). Our observations confirm and extend the decreasing trend of the $[\mathrm{N}$ II]/FIR ratio as a function of the continuum luminosity, toward high luminosities from local galaxies with $[\mathrm{N} \mathrm{II}]_{122} \mu \mathrm{m} / \mathrm{FIR} \sim 3 \times 10^{-4}$ (Malhotra et al. 2001; Panuzzo et al. 2010; Fischer et al. 2010; Edge et al. 2010; Beirão et al. 2010; Vasta et al. 2010; Garciá-Carpio et al. 2011)

\footnotetext{
${ }^{17}$ Here we consider the total FIR luminosity. However, Weiß et al. (2007) modeled the FIR emission in APM 08279+5255 with two components,

"warm" and "cold." If the former is powered from the quasar instead of star formation, the FIR luminosity should be scaled to $10 \%$ of the adopted value.
} 

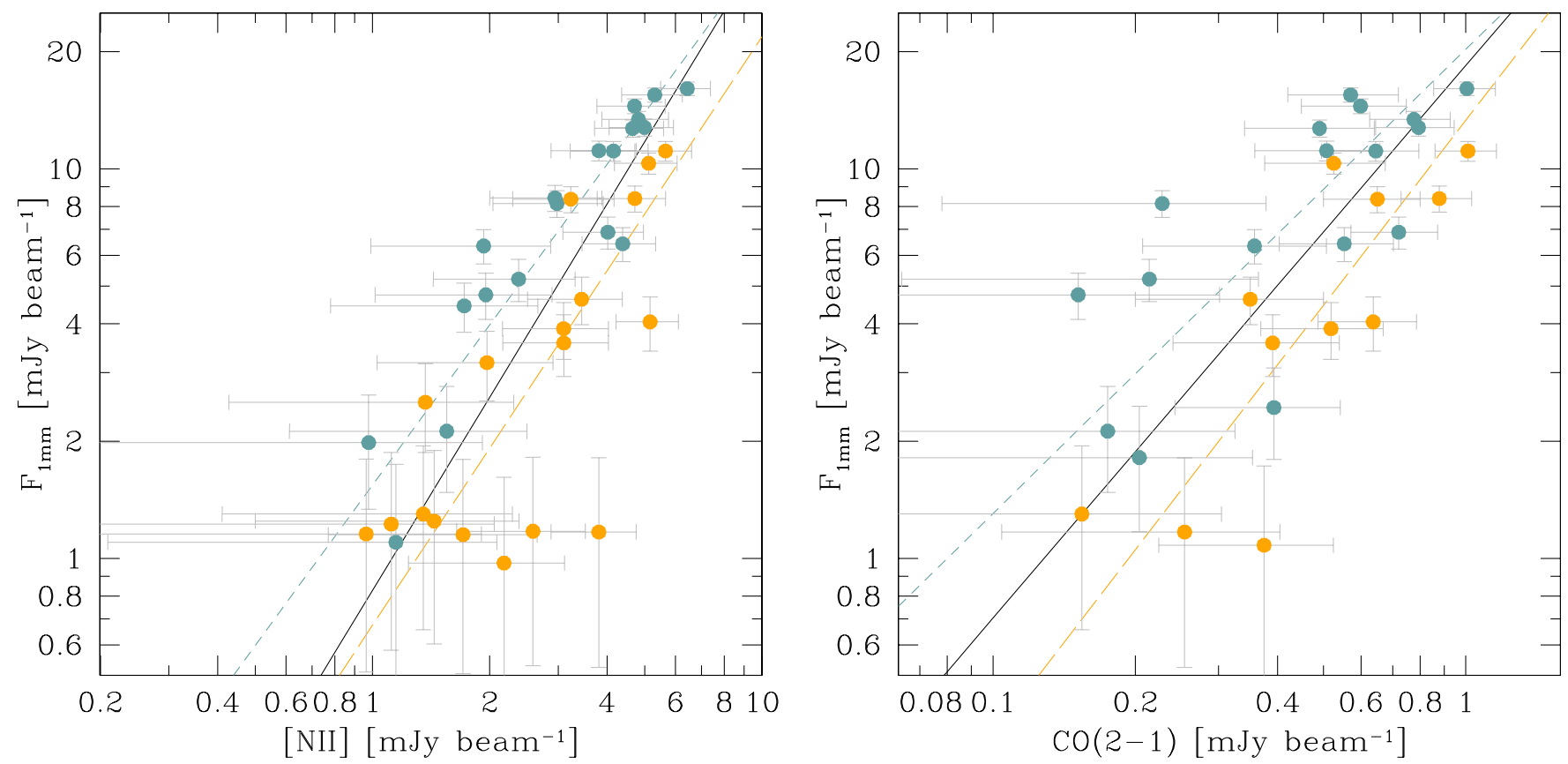

Figure 4. Comparison between the FIR continuum surface brightness at $1 \mathrm{~mm}$ (observed frame) and the surface brightness of $[\mathrm{N} \mathrm{II}]_{205} \mu \mathrm{m}(\mathrm{left}$ panel) and CO(2-1) (right panel) in our PdBI and EVLA observations of MM 18423+5938. Since the FIR surface brightness can be used as a proxy for the surface density of star formation $\left(\Sigma_{\mathrm{SFR}}\right)$, while the CO surface brightness maps the surface density of molecular gas $\left(\Sigma_{\mathrm{H}_{2}}\right)$, the right panel shows the first, spatially resolved star formation law in a high- $z$ galaxy. Each point is a 0.'5 $50^{\prime \prime} 5$ pixel with $>1 \sigma$ flux. Error bars show the corresponding $1 \sigma$ uncertainties. Gray (orange) points refer to the northern (southern) part of the Einstein ring, with a cut at declination=+59:38:29.3. The FIR emission increases with the $\left[\mathrm{N} \mathrm{III}_{205 \mu \mathrm{m}}\right.$ and $\operatorname{CO}(2-1)$ emission $\left(\Sigma_{\mathrm{F}_{1 \mathrm{~mm}}} \propto \Sigma_{\mathrm{N}}^{1.4}\right.$ and $\left.\Sigma_{\mathrm{F}_{1 \mathrm{~mm}}} \propto \Sigma_{\mathrm{CO}}^{1.6}\right)$; best fits on the total, northern and southern pixels are shown as black solid, gray short-dashed, and orange long-dashed lines, respectively. The FIR continuum is $\sim 2$

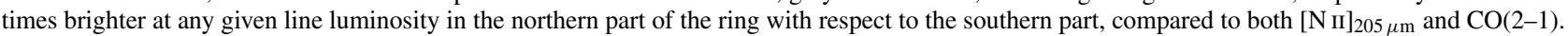

(A color version of this figure is available in the online journal.)



Figure 5. $\left[\mathrm{N} \mathrm{II}_{122} \mu \mathrm{m} /\right.$ FIR luminosity ratio, as a function of the FIR continuum luminosity. When the $122 \mu \mathrm{m}$ line is not available (like in the sources presented here), we assumed a [N II] 122-205 $\mu$ m luminosity ratio of 5 (see, e.g., Beirão et al. 2010). High-redshift sources (filled symbols) are labeled. The horizontal bars mark the uncertainties in the magnification correction of our sources $(4-100$ for APM 08279+5255, see Weiß et al. 2007; Riechers et al. 2009; 12-20 for MM 18423+5938, see Lestrade et al. 2011).

(A color version of this figure is available in the online journal.) to FIR-luminous sources with $[\mathrm{N} \mathrm{II}]_{122 \mu \mathrm{m}} / \mathrm{FIR} \sim 3-5 \times 10^{-5}$. Even in the extreme case of a 122 -to- $205 \mu \mathrm{m}$ ratio of 10 , our data would populate a very different region of the plot with respect to the values found by Ferkinhoff et al. (2011), who reported high $[\mathrm{N} \text { II }]_{122 \mu \mathrm{m}} /$ FIR ratios $\left(\sim 10^{-3}\right)$ in two FIR-bright sources.

\section{CONCLUSIONS}

We present a study of the ionized ISM in two high- $z$ sources based on the forbidden ionized nitrogen emission line at $205 \mu \mathrm{m}$. The FIR transitions of ionized nitrogen represent extremely powerful tools to study the properties of purely ionized gas in distant galaxies. A larger number of $[\mathrm{N} \mathrm{II}]_{205} \mu \mathrm{m}$ detections in distant galaxies is now mandatory in order to build a suitable sample for statistical analysis. This is now possible thanks to the technological upgrades in the field. In particular, the unparalleled sensitivities reached by ALMA will open new possibilities for the studies of high- $z$ objects.

We thank the anonymous referee for his/her expert comments that increased the quality of the manuscript. We thank B. Groves for useful discussions. This work is based on observations carried out with the IRAM Plateau de Bure Interferometer. IRAM is supported by INSU/CNRS (France), MPG (Germany), and IGN (Spain). R.D. acknowledges funding from Germany's National Research Centre for Aeronautics and Space (DLR, project FKZ 50 OR 1104). D.R. acknowledges support from NASA through a Spitzer Space Telescope grant. 


\section{REFERENCES}

Beirão, P., Armus, L., Appleton, P. N., et al. 2010, A\&A, 518, L60 Benford, D. J. 1999, PhD thesis, Caltech

Bigiel, F., Leroy, A., Walter, F., et al. 2008, AJ, 136, 2846

Bradford, C. M., Bolatto, A. D., Maloney, P. R., et al. 2011, ApJ, 741, L37

Colgan, S. W. J., Haas, M. R., Erickson, E. F., et al. 1993, ApJ, 413, 237

Combes, F., Rex, M., Rawle, T. D., et al. 2012, A\&A, 538, L4

Cox, P., Krips, M., Neri, R., et al. 2011, ApJ, 740, 63

Daddi, E., Elbaz, D., Walter, F., et al. 2010, ApJ, 714, L118

De Breuck, C., Maiolino, R., Caselli, P., et al. 2011, A\&A, 530, L8

Downes, D., Neri, R., Wiklind, T., Wilner, D. J., \& Shaver, P. A. 1999, ApJ, 513, L1

Edge, A. C., Oonk, J. B. R., Mittal, R., et al. 2010, A\&A, 518, L46

Egami, E., Neugebauer, G., Soifer, B. T., et al. 2000, ApJ, 535, 561

Ferkinhoff, C., Brisbin, D., Nikola, T., et al. 2011, ApJ, 740, L29

Ferkinhoff, C., Hailey-Dunsheath, S., Nikola, T., et al. 2010, ApJ, 714, L147

Fischer, J., Sturm, E., González-Alfonso, E., et al. 2010, A\&A, 518, L41

Garciá-Carpio, J., Sturm, E., Hailey-Densheath, S., et al. 2011, ApJ, 728, L7

Genzel, R., Tacconi, L. J., Gracia-Carpio, J., et al. 2010, MNRAS, 407, 2091

Iono, D., Yun, M. S., Elvis, M., et al. 2006, ApJ, 645, L97

Ivison, R. J., \& Harrison, A. P. 1996, A\&A, 309, 416

Ivison, R. J., Swinbank, A. M., Swinyard, B., et al. 2010, A\&A, 518, L35

Kennicutt, R. C. 1998, ApJ, 498, 541

Krips, M., Peck, A. B., Sakamoto, K., et al. 2007, ApJ, 671, L5

Leroy, A. K., Walter, F., Brinks, E., et al. 2008, AJ, 136, 2782

Lestrade, J.-F., Carilli, C. L., Thanjavur, K., et al. 2011, ApJ, 739, L30

Lestrade, J.-F., Combes, F., Salomé, P., et al. 2010, A\&A, 522, L4
Loenen, A. F., van der Werf, P. P., Güsten, R., et al. 2010, A\&A, 521, L2

Luhman, M. L., Satyapal, S., Fischer, J., et al. 1998, ApJ, 504, L11

Madden, S. C., Poglitsch, A., Geis, N., Stacey, G. J., \& Townes, C. H. 1997, ApJ, 483, 200

Maiolino, R., Caselli, P., Nagao, T., et al. 2009, A\&A, 500, L1

Maiolino, R., Cox, P., Caselli, P., et al. 2005, A\&A, 440, L51

Malhotra, S., Kaufman, M. J., Hollenbach, D., et al. 2001, ApJ, 561, 766

Matteucci, F., \& Padovani, P. 1993, ApJ, 419, 485

Oberst, T. E., Parshley, S. C., Stacey, G. J., et al. 2006, ApJ, 652, L125

Panuzzo, P., Rangwala, N., Rykala, A., et al. 2010, A\&A, 518, L37

Riechers, D. A., Walter, F., Carilli, C. L., \& Lewis, G. F. 2009, ApJ, 690, 463

Savage, B. D., \& Sembach, K. R. 1996, ARA\&A, 34, 279

Schruba, A., Leroy, A. K., Walter, F., et al. 2011, AJ, 142, 37

Solomon, P. M., Downes, D., \& Radford, S. J. E. 1992, ApJ, 398, L29

Stacey, G. J., Geis, N., Genzel, R., et al. 1991, ApJ, 373, 423

Stacey, G. J., Hailey-Dunsheath, S., Ferkinhoff, C., et al. 2010, ApJ, 724, 957

Valtchanov, I., Virdee, J., Ivison, R. J., et al. 2011, MNRAS, 415, 3473

van der Werf, P. P., Isaak, K. G., Meijerink, R., et al. 2010, A\&A, 518, L42

Vasta, M., Barlow, M. J., Viti, S., Yates, J. A., \& Bell, T. A. 2010, MNRAS, 404, 1910

Wagg, J., Carilli, C. L., Wilner, D. J., et al. 2010, A\&A, 519, L1

Wagg, J., Wilner, D. J., Neri, R., Downes, D., \& Wiklind, T. 2006, ApJ, 651, 46

Walter, F., Riechers, D., Cox, P., et al. 2009a, Nature, 457, 699

Walter, F., Weiss, A., Downes, D., Decarli, R., \& Henkel, C. 2011, ApJ, 730, 18

Walter, F., Weiss, A., Riechers, D. A., et al. 2009b, ApJ, 691, L1

Weiß, A., Downes, D., Neri, R., et al. 2007, A\&A, 467, 955

White, G. J., Abergel, A., Spencer, L., et al. 2010, A\&A, 518, L114

Wong, T., \& Blitz, L. 2002, ApJ, 569, 157

Wright, E. L., Mather, J. C., Bennett, C. L., et al. 1991, ApJ, 381, 200 